\title{
Adaptive COVID-19 Forecasting via Bayesian Optimization
}

\author{
Nayana Bannur \\ Wadhwani AI \\ India
}

nayana.bannur@wadhwaniai.org

\author{
Shreyas Shetty \\ Flipkart Internet Pvt. Ltd. \\ India \\ shreyas.shetty@flipkart.com
}

\author{
Harsh Maheshwari \\ Flipkart Internet Pvt. Ltd. \\ India \\ harsh.maheshwari@flipkart.com \\ Srujana Merugu
Independent
India
srujana@gmail.com
}

\author{
Sansiddh Jain \\ Wadhwani AI \\ India \\ sansiddh@wadhwaniai.org \\ Alpan Raval
Wadhwani AI
India
alpan@wadhwaniai.org
}

\begin{abstract}
Accurate forecasts of infections for localized regions are valuable for policy making and medical capacity planning. Existing compartmental and agent-based models for epidemiological forecasting employ static parameter choices and cannot be readily contextualized, while adaptive solutions focus primarily on the reproduction number. The current work proposes a novel model-agnostic Bayesian optimization approach for learning model parameters from observed data that generalizes to multiple application-specific fidelity criteria. Empirical results point to the efficacy of the proposed method with SEIR-like models on COVID-19 case forecasting tasks. A city-level forecasting system based on this method is being used for COVID-19 response in a few impacted Indian cities.
\end{abstract}

\section{ACM Reference Format:}

Nayana Bannur, Harsh Maheshwari, Sansiddh Jain, Shreyas Shetty, Srujana Merugu, and Alpan Raval. 2021. Adaptive COVID-19 Forecasting via Bayesian Optimization. In 8th ACM IKDD CODS and 26th COMAD (CODS COMAD 2021), fanuary 2-4, 2021, Bangalore, India. ACM, New York, NY, USA, 1 page. https://doi.org/10.1145/3430984.3431047

\section{INTRODUCTION}

The ongoing COVID-19 pandemic and the consequent devastating increase in morbidity and mortality have accentuated the need for robust epidemiological forecasting models. Deployment of such models as part of the public health response requires support for (a) fine-grained contextualization to account for spatio-temporal variations in contact behaviour, lockdown, testing, hospitalization, and reporting policies, (b) multiple models depending on the case count availability (e.g., age-stratified or testing-based extensions), (c) addressing varying data reliability due to reporting delays, and (d) multiple application use cases with different fidelity requirements (e.g., medical preparedness is tied to accurate $2-4$ week forecasts while long-term policy making might focus on peak estimation). Most existing models [4] that use static parameters from domain knowledge and even adaptive likelihood maximization-based methods [5] do not adequately address these requirements.

Permission to make digital or hard copies of part or all of this work for personal or classroom use is granted without fee provided that copies are not made or distributed for profit or commercial advantage and that copies bear this notice and the full citation on the first page. Copyrights for third-party components of this work must be honored For all other uses, contact the owner/author(s).

CODS COMAD 2021, fanuary 2-4, 2021, Bangalore, India

(c) 2021 Copyright held by the owner/author(s).

ACM ISBN 978-1-4503-8817-7/21/01.

https://doi.org/10.1145/3430984.3431047
Problem Statement: For $t \in\left[0, t_{c u r r}\right]$ and region $r$, given the case count time series $\mathbf{x}(t, r)$ and region metadata $\mathbf{w}(r)$ (e.g. population), forecast $\mathbf{x}_{\text {pred }}(t, r)$ for $t \in\left[t_{\text {curr }}, t_{c u r r}+d\right]$ s.t. an application specific loss $L\left(\mathbf{x}_{\text {pred }}(:, r), \mathbf{x}(:, r)\right)$ on the forecast period is minimized.

\section{PROPOSED SOLUTION}

BayesOpt-based Blackbox Learning: For any parametric forecasting model of the form $f_{\theta}(\mathbf{x}(t), d)=\mathbf{x}_{\text {pred }}(t+1: t+d)$ we optimize $\theta^{*}=\operatorname{argmin}_{\theta} L\left(f_{\theta}\left(\mathbf{x}\left(t^{\prime}\right), d\right), \mathbf{x}\left(t^{\prime}+1: t^{\prime}+d\right)\right)$ using observations from the period $\left[t^{\prime}, t^{\prime}+d\right]$ for an appropriate loss function $L(\cdot)$. Optimizers such as the hyperopt library [3] can be used.

Uncertainty Estimation: Since certain applications require confidence intervals, the parameter sets (or trials) explored during Bayesian optimization are used to construct a posterior distribution $p\left(f_{\theta}(x) \mid D\right)$ on the parameter space given data $D$ via a mapping from the observed loss values $L(\cdot)$ and the generative distribution. For instance, in case of exponential families [2], the posterior probability $p\left(f_{\theta}(x) \mid D\right) \propto \exp \left(-c L\left(f_{\theta}(x), D\right)\right.$ and $c$ is estimated via validation on a holdout period.

Model Class \& Initial Conditions: For practical deployment, we chose SEIR extensions due to their parsimonious nature, flexibility to incorporate testing effects and stratification, and high interpretability. While observed compartments can be readily initialized, the initial values of unobserved compartments (e.g., exposed) are viewed as latent variables and estimated similar to other model parameters, thus also partially accounting for imported cases.

Experiments: Appropriate choices (e.g., training duration) for the Bayesian optimization were identified via extensive exploration. The efficacy and flexibility of the proposed method when applied to SEIR models was evaluated relative to other baselines on both real and synthetic case data. Typically, the ensemble-mean provides a stable forecast (mean absolute percentage error $<10 \%$ ) [1].

\section{REFERENCES}

[1] Nayana Bannur, Harsh Maheshwari, Sansiddh Jain, Shreyas Shetty, Srujana Merugu, and Alpan Raval. 2020. Adaptive COVID-19 Forecasting via Bayesian Optimization. medRxiv (2020). https://doi.org/10.1101/2020.10.19.20215293 arXiv:https://www.medrxiv.org/content/early/2020/10/27/2020.10.19.20215293

[2] O.E. Barndorff-Nielsen. 1978. Information and Exponential Families: In Statistical Theory. Wiley. https://books.google.co.in/books?id=QxbvAAAAMAAJ

[3] James Bergstra, Rémi Bardenet, Yoshua Bengio, and Balázs Kégl. 2011. Algorithms for Hyper-Parameter Optimization. In Proceedings of the 24th International Conference on Neural Information Processing Systems (Granada, Spain) (NIPS'11). Curran Associates Inc., Red Hook, NY, USA, 2546-2554.

[4] Herbert W. Hethcote. 2000. The Mathematics of Infectious Diseases. SIAM Rev. 42, 4 (Dec. 2000), 599-653. https://doi.org/10.1137/S0036144500371907

[5] Kevin Systrom, Thomas Vladek, and Mike Krieger. 2020. Rt COVID-19. https: //github.com/rtcovidlive/covid-model. 